\title{
The ultraluminous x-ray sources ngc 1313 x-1 and x-2: a broadband study with
} NuSTAR and XMM-Newton

Bachetti, Matteo; Rana, Vikram; Walton, Dominic J.; Barret, Didier; Harrison, Fiona A.; Boggs, Steven E.; Christensen, Finn Erland; Craig, William W.; Fabian, Andrew C.; Fuerst, Felix

Total number of authors:

19

Published in:

Astrophysical Journal

Link to article, DOI:

10.1088/0004-637X/778/2/163

Publication date:

2013

Document Version

Publisher's PDF, also known as Version of record

Link back to DTU Orbit

Citation (APA):

Bachetti, M., Rana, V., Walton, D. J., Barret, D., Harrison, F. A., Boggs, S. E., Christensen, F. E., Craig, W. W., Fabian, A. C., Fuerst, F., Grefenstette, B. W., Hailey, C. J., Hornschemeier, A., Madsen, K. K., Miller, J. M.,

Ptak, A. F., Stern, D., Webb, N. A., \& Zhang, W. W. (2013). The ultraluminous $x$-ray sources ngc $1313 \mathrm{x}-1$ and $\mathrm{x}$ 2: a broadband study with NuSTAR and XMM-Newton. Astrophysical Journal, 778(2).

https://doi.org/10.1088/0004-637X/778/2/163

\section{General rights}

Copyright and moral rights for the publications made accessible in the public portal are retained by the authors and/or other copyright owners and it is a condition of accessing publications that users recognise and abide by the legal requirements associated with these rights.

- Users may download and print one copy of any publication from the public portal for the purpose of private study or research.

- You may not further distribute the material or use it for any profit-making activity or commercial gain

- You may freely distribute the URL identifying the publication in the public portal 


\title{
THE ULTRALUMINOUS X-RAY SOURCES NGC 1313 X-1 AND X-2: A BROADBAND STUDY WITH NuSTAR AND XMM-Newton
}

\author{
Matteo Bachetti ${ }^{1,2}$, Vikram Rana ${ }^{3}$, Dominic J. Walton ${ }^{3}$, Didier Barret ${ }^{1,2}$, Fiona A. Harrison ${ }^{3}$, Steven E. Boggs ${ }^{4}$, \\ Finn E. Christensen ${ }^{5}$, William W. Craig ${ }^{4}, 6$, Andrew C. Fabian ${ }^{7}$, Felix Fürst ${ }^{3}$, Brian W. Grefenstette ${ }^{3}$, \\ Charles J. Haile ${ }^{8}$, Ann Hornschemeier ${ }^{9}$, Kristin K. Madsen ${ }^{3}$, Jon M. Miller ${ }^{10}$, Andrew F. Ptak ${ }^{9}$, \\ Daniel Stern ${ }^{11}$, Natalie A. WebB ${ }^{1,2}$, and William W. Zhang ${ }^{9}$ \\ ${ }^{1}$ Université de Toulouse, UPS-OMP, IRAP, Toulouse, France; matteo.bachetti@irap.omp.eu \\ ${ }^{2}$ CNRS, Institut de Recherche en Astrophysique et Planétologie, 9 Av. Colonel Roche, BP 44346, F-31028 Toulouse Cedex 4, France \\ ${ }^{3}$ Cahill Center for Astronomy and Astrophysics, Caltech, Pasadena, CA 91125, USA \\ ${ }^{4}$ Space Sciences Laboratory, University of California, Berkeley, CA 94720, USA \\ ${ }^{5}$ DTU Space, National Space Institute, Technical University of Denmark, Elektrovej 327, DK-2800 Lyngby, Denmark \\ ${ }^{6}$ Lawrence Livermore National Laboratory, Livermore, CA 94550, USA \\ ${ }^{7}$ Institute of Astronomy, University of Cambridge, Madingley Road, Cambridge CB3 0HA, UK \\ ${ }^{8}$ Columbia Astrophysics Laboratory, Columbia University, New York, NY 10027, USA \\ ${ }^{9}$ NASA Goddard Space Flight Center, Greenbelt, MD 20771, USA \\ ${ }^{10}$ Department of Astronomy, University of Michigan, 500 Church Street, Ann Arbor, MI 48109-1042, USA \\ ${ }^{11}$ Jet Propulsion Laboratory, California Institute of Technology, Pasadena, CA 91109, USA \\ Received 2013 July 25; accepted 2013 October 2; published 2013 November 13
}

\begin{abstract}
We present the results of NUSTAR and XMM-Newton observations of the two ultraluminous X-ray sources: NGC $1313 \mathrm{X}-1$ and X-2. The combined spectral bandpass of the two satellites enables us to produce the first spectrum of X-1 between 0.3 and $30 \mathrm{keV}$, while X-2 is not significantly detected by NuSTAR above $10 \mathrm{keV}$. The NuSTAR data demonstrate that X-1 has a clear cutoff above $10 \mathrm{keV}$, whose presence was only marginally detectable with previous X-ray observations. This cutoff rules out the interpretation of X-1 as a black hole in a standard low/hard state, and it is deeper than predicted for the downturn of a broadened iron line in a reflection-dominated regime. The cutoff differs from the prediction of a single-temperature Comptonization model. Further, a cold disklike blackbody component at $\sim 0.3 \mathrm{keV}$ is required by the data, confirming previous measurements by XMM-Newton only. We observe a spectral transition in X-2, from a state with high luminosity and strong variability to a lowerluminosity state with no detectable variability, and we link this behavior to a transition from a super-Eddington to a sub-Eddington regime.
\end{abstract}

Key words: accretion, accretion disks - black hole physics - stars: black holes - X-rays: individual (NGC $1313 \mathrm{X}-1$, NGC 1313 X-2) - X-rays: stars

Online-only material: color figures

\section{INTRODUCTION}

Ultraluminous X-ray sources (ULXs) are off-nuclear pointlike sources with apparent X-ray luminosities exceeding the Eddington limit for stellar-mass black holes (StMBHs). Their high luminosity can be due to yet-unknown mechanisms of super-Eddington accretion on a StMBH (or beamed emission from it) or the presence of a black hole (BH) with a high mass, such as an intermediate-mass black hole (IMBH). While for luminosities $>10^{41} \mathrm{erg} \mathrm{s}^{-1}$ the identification with an IMBH is most probable, as was shown for the source HLX-1 (Farrell et al. 2009), for lower luminosities both mechanisms can apply. Convincing evidence for super-Eddington accretion has been reported for two ULXs in M31 (Middleton et al. 2012, 2013). See Roberts (2007) and Feng \& Soria (2011) for reviews.

ULX spectra below $10 \mathrm{keV}$ have been thoroughly investigated (see, e.g., Gladstone et al. 2009) with XMM-Newton (Jansen et al. 2001), Suzaku (Mitsuda et al. 2007), and Chandra (Weisskopf et al. 2002). Their X-ray spectral shape does not match that of known $\mathrm{BHs}$, in the mass range from 10 to millions of solar masses (see Done et al. 2007 for a review of standard BHs). A spectral break below $10 \mathrm{keV}$ has been observed in most ULXs (Stobbart et al. 2006; Gladstone et al. 2011), together with a disk-like blackbody component at low temperatures $(\lesssim 0.3 \mathrm{keV})$. This latter component, if produced by a standard disk reaching the proximity of the $\mathrm{BH}$, would imply masses above $\sim 100 M_{\odot}$, and thus the presence of an IMBH (Miller et al. 2003, 2004). But the temperature-luminosity relation for this component does not match that expected in standard accretion disks in the soft state, where the disks extend to the innermost stable circular orbit (see, e.g., Kajava \& Poutanen 2009; Feng \& Soria 2011 for a review). This relation can be partially recovered in some cases by assuming a constant absorption column between the observations (Miller et al. 2013) or using non-standard disk models (Vierdayanti et al. 2006). Also, the cutoff is at much lower temperature than is expected in standard BH hard states (Done et al. 2007). Some authors associate the low-temperature disk-like component with the presence of an optically thick corona that blocks the inner part of the disk, so that the visible part of the disk has a much lower temperature (Gladstone et al. 2009). Others suggest that it might come from a strong outflow (e.g., King 2004) or be the result of blurred line emission from highly ionized, fast-moving gas (Gonçalves $\&$ Soria 2006).

From X-ray data below $10 \mathrm{keV}$ it is impossible to distinguish between a cutoff and a downturn produced by the imperfect fit of the continuum due to the presence of a broadened iron complex in a reflection-dominated regime (Caballero-Garcia \& Fabian 2010; Gladstone et al. 2011). The difference becomes clear above $10 \mathrm{keV}$ (see, e.g., Walton et al. 2011a), in a region of the spectrum that past sensitive satellites could not explore. 
Table 1

Summary of the Data Used in This Paper

\begin{tabular}{|c|c|c|c|}
\hline Camera & $\begin{array}{c}\text { Exposure } \\
(\mathrm{ks})\end{array}$ & X-1 Counts & X-2 Counts \\
\hline \multicolumn{4}{|c|}{ Epoch $1-2012$ Dec 16} \\
\hline FPMA & 100.9 & $3314(386.2)$ & $2336(1074.2)$ \\
\hline FPMB & 100.8 & $3444(473.0)$ & $2504(1076.3)$ \\
\hline EPIC-pn & 93.8 & $74002(1523.4)$ & $52603(1029.1)$ \\
\hline EPIC-MOS $1^{\mathrm{a}}$ & 114.5 & 27785 (309.2) & $21233(318.8)$ \\
\hline EPIC-MOS2 & 115.1 & $29917(339.5)$ & $21304(323.6)$ \\
\hline \multicolumn{4}{|c|}{ Epoch $2-2012$ Dec 21-22 } \\
\hline FPMA & 127.0 & $4166(472.9)$ & $1898(1333.3)$ \\
\hline FPMB & 127.0 & $4237(584.2)$ & $1918(1517.4)$ \\
\hline EPIC-pn ${ }^{\mathrm{b}}$ & 79.2 & 70605 (881.6) & $22925(759.2)$ \\
\hline EPIC-MOS $1^{a}$ & 116.0 & 20439 (411.4) & $12378(384.8)$ \\
\hline EPIC-MOS2 & 121.8 & $32796(439.6)$ & $13593(326.8)$ \\
\hline
\end{tabular}

Notes. Values in parentheses are background counts, scaled to the source region size.

a $\mathrm{X}-1$ on detector edge.

b $\mathrm{X}-2$ on detector edge.

The Nuclear Spectroscopic Telescope Array (NuSTAR; Harrison et al. 2013), launched in 2012 June, with its focusing capabilities, large bandpass between 3 and $80 \mathrm{keV}$ and large effective area, represents the ideal complement to XMM-Newton (given the similar effective area between 5 and $10 \mathrm{keV}$ and spectral capabilities). X-rays are focused by multilayer-coated grazing incidence optics onto two independent focal plane modules, called Focal Plane Module A and B (hearafter FPMA and FPMB). Each focal plane contains four cadmium zinc telluride detectors. The spatial resolution is $58^{\prime \prime}$ half-power diameter and $18^{\prime \prime}$ FWHM. NuSTAR is therefore a powerful tool for studying ULX broadband X-ray spectra. Since the launch of the satellite, we have observed a sample of luminous $\left(L_{\mathrm{x}} \sim 10^{40} \mathrm{erg} \mathrm{s}^{-1}\right)$, close-by ( $d \lesssim 10 \mathrm{Mpc}$ ), and hard (showing X-ray power-law photon index $\Gamma \lesssim 2$ below $10 \mathrm{keV}$ ) ULXs simultaneously with NUSTAR and Suzaku or XMM-Newton, producing the first ULX spectra extending over the range $0.3-30 \mathrm{keV}$.

In this paper, we describe the results obtained for the two ULXs in the spiral galaxy NGC 1313 ( $d \sim 4.13 \mathrm{Mpc}$; Méndez et al. 2002). These two ULXs are among the brightest, hardest, and closest ULXs (Swartz et al. 2004; Walton et al. 2011b), and therefore they are ideal targets for our program. They are known to show spectral variability below $10 \mathrm{keV}$ (Feng \& Kaaret 2006; Dewangan et al. 2010; Pintore \& Zampieri 2012). Significant variability at high fluxes has also been observed in both sources (Heil et al. 2009).

In Section 2 we describe the observations done, in Section 3 we provide some details on data reduction, then in Sections 4 and 5 we discuss the spectral and timing analysis of the two sources, and finally in Section 6 we discuss the results.

\section{THE OBSERVATIONS}

During this campaign, we observed NGC 1313 with $X M M-N e w t o n$ and NUSTAR two times, as summarized in Table 1. Observations were executed with a separation of about a week, to search for variability. The two ULXs are separated by about $7^{\prime}$ and can be observed simultaneously by XMM-Newton and NuSTAR. We chose to place X-1 close to the optical axis. It was not possible to keep both ULXs close to the optical axis of
$N u S T A R$, so we chose to obtain the best spectral quality for at least one of them rather than reducing the quality for both. X-1 is historically brighter and harder than X-2 (Pintore \& Zampieri 2011), and we estimated that the addition of NuSTAR data would yield more valuable new information for this source.

\section{DATA REDUCTION}

\subsection{NuSTAR Data}

NUSTAR data were processed using the version 1.0.1 of the NUSTAR data analysis system, (NUSTAR DAS). The NuSTAR $D A S$ tools are divided in two main parts: the preprocessing pipeline (nupipeline) that produces the L1 filtered files, and the products pipeline (nuproducts) that is used to extract spectra, light curves, and other high-level products.

We ran nupipeline on all observations with the default options for good time interval filtering and produced cleaned event files. We then ran nuproducts using a 30" extraction region around X-1 (see Section 3.1 for the details) and a $60^{\prime \prime}$ extraction region around X-2, and for background an $80^{\prime \prime}$ extraction region in the same detector as the source, further than $1^{\prime}$ away to avoid contributions from the point-spread function (PSF) wings. We applied standard PSF, alignment, and vignetting corrections. Spectra were rebinned in order to have at least 20 counts bin $^{-1}$ to ensure the applicability of the $\chi^{2}$ statistics, and in some cases to 50 counts bin ${ }^{-1}$ in order to reduce computation times in particularly complicated models.

As it turned out, NuSTAR data of X-2 produced very poor spectral information above $\sim 10 \mathrm{keV}$. Besides being very faint, the NuSTAR data were likely to be affected by response degradation due to the off-axis position of the source, and a very uncertain background level due to the NUSTAR sloping aperture background. We decided not to use them for the next steps of the analysis.

As can be seen in Figure 1, NGC 1313 X-1 has a nearby contaminating source separated by $\sim 53^{\prime \prime}$ that is not clearly resolved by NuSTAR. While the source is outside the XMM-Newton PSF of X-1 and it is quite easy to avoid it through the choice of a small extraction region, the evaluation of its possible effect on $N U S T A R$ data is less straightforward, due to the larger PSF. The contaminating source has a flux $\sim 10$ times lower than X-1 in the $X M M-N e w t o n$ band, but the NuSTAR PSF of X-1 appears elongated toward the contaminating source. To evaluate the effects of this source, we produced NUSTAR spectra with two different extraction regions, one including the nearby source (radius $80^{\prime \prime}$ ) and one not including it (radius 30"). As shown in Figure 2, the two spectra do not differ substantially between $10 \mathrm{keV}$ and the intersection of source and background levels, while there is some minor deviation at lower energy, in the XMM-Newton band. The best-fit power laws below $10 \mathrm{keV}$ in the two data sets are marginally compatible (spectral index $2.08 \pm 0.08$ in the first and $1.9 \pm 0.1$ in the second; quoted errors are $90 \%$ confidence limits). We chose to use the smaller extraction region for precaution. This analysis shows that the residual effect is negligible if the $30^{\prime \prime}$ extraction region is used. In the following analysis, we only consider spectra below $30 \mathrm{keV}$ where the source is stronger than, or compatible with, the background.

\subsection{XMM-Newton Data}

The XMM-Newton data reduction was carried out with the $X M M-N e w t o n$ Science Analysis System (SAS v12.0.1). We produced calibrated event files with epproc and emproc, created 

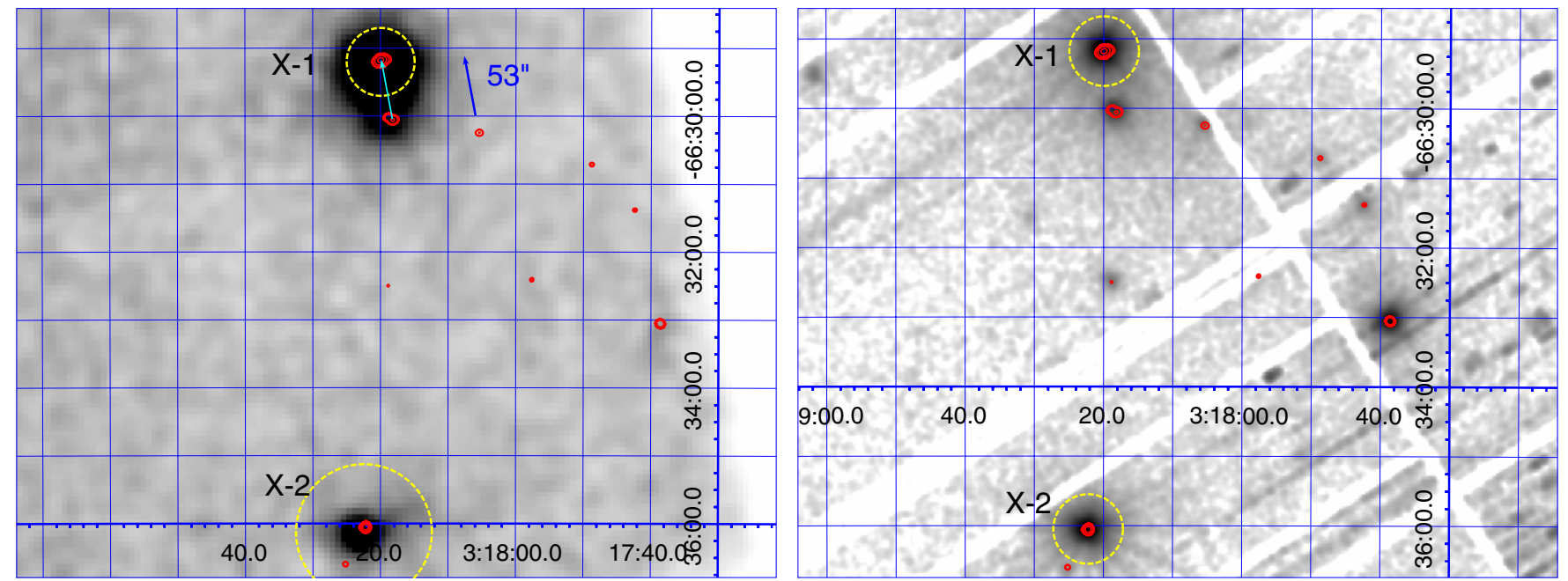

Figure 1. (Left) NuSTAR and (right) EPIC-pn images of the two ULXs, produced with DS9 (Joye \& Mandel 2003). Data are from all energy bands of the detectors. Chandra contours corresponding to the ULXs and possible contaminants are shown in red. Yellow dashed regions are the extraction regions used for analysis. The radius of the region around X-1 is $30^{\prime \prime}$ in both cases in order to avoid the contaminating source about $50^{\prime \prime} \mathrm{SE}$ of the source. For X-2, instead, it is $60^{\prime \prime}$ in $N u S T A R$ and $30^{\prime \prime}$ for XMM-Newton.

(A color version of this figure is available in the online journal.)

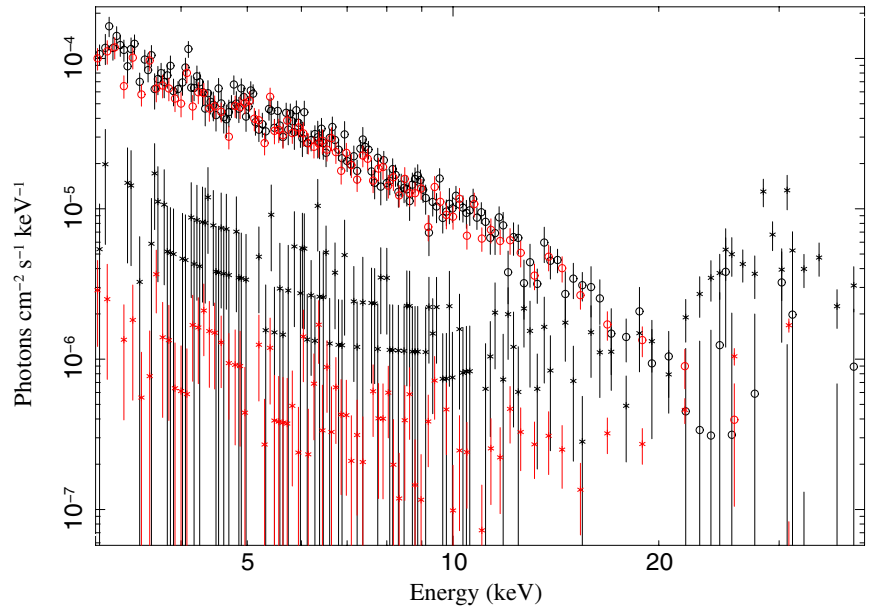

Figure 2. NUSTAR spectrum of $\mathrm{X}-1$, rebinned to 30 counts bin ${ }^{-1}$; black corresponds to an $80^{\prime \prime}$ extraction region, red to $30^{\prime \prime}$ (which excludes the contaminating region $53^{\prime \prime} \mathrm{SE}$ of X-1). Circles label the source spectra, while " $\mathrm{x}$ "s mark the background spectra. There is no significant change in the spectrum between 10 and $30 \mathrm{keV}$, but the larger extraction region is much more affected by background. Also, below $10 \mathrm{keV}$ there is some very small deviation.

(A color version of this figure is available in the online journal.)

custom good time interval files to filter out periods of high background according to the prescription in the SAS manual, and selected only \#XMMEA_EP \&\& PATTERN $<4$ events for EPIC-pn and \#XMMEA_EM \&\& PATTERN $<12$ events for EPIC-MOS cameras. We also filtered the events along detector gaps through $F L A G==0$. The resulting event files were then filtered with a $30^{\prime \prime}$ region around the two ULXs. Background events were selected in each detector in regions with no detector edges, bad pixels, or visible sources.

Spectra were extracted for all three cameras, unless the source was in a detector gap (see Table 1). We used fselect for spectral extraction, and ancillary responses and redistribution matrices were created with arfgen and rmfgen, with the new ELLBETA PSF correction enabled. Spectra were finally rebinned with grppha in order to have at least 20 counts bin ${ }^{-1}$.

\section{SPECTRAL ANALYSIS}

\subsection{Software Tools and General Procedure}

Spectral analysis was carried out with the Interactive Spectral Analysis System (ISIS; Houck \& Denicola 2000). We chose this software over the more commonly used X-ray spectral fitting package XSPEC (Arnaud 1996) because of its scriptability and the transparent use (in multicore computers) of parallel processing during confidence region calculation and parameter space searching, while being able to use all XSPEC models, table and local models. ${ }^{12}$

To model neutral absorption we used the tbnew model,,${ }^{13}$ the new version of tbabs (Wilms et al. 2000) featuring higher spectral resolution and, also importantly, much faster computation due to caching techniques (see linked Web site for the details). This model can be used in different ways by including custom abundances of a large number of elements. We use the simplest version, tbnew_feo, including only the abundances of iron and oxygen besides the usual hydrogen column $n_{\mathrm{H}}$, and fixing the abundances of all elements to the standard values from Wilms et al. (2000). We use the cross sections from Verner et al. (1996). The hydrogen column we measure from our fits is at least $\sim 5$ times higher than the Galactic values taken from Kalberla et al. (2005). Therefore for simplicity we use only one component for modeling absorption instead of the two that would be necessary were the values comparable.

When jointly fitting NUSTAR and XMM-Newton data, we first fit a constant*cut offpl model between 5 and $10 \mathrm{keV}$, with the constant for EPIC detectors fixed to 1 and the others left

\footnotetext{
${ }^{12}$ In the following sections we will show several unfolded spectra (i.e., spectra corrected for the response and thus ideally equal to the "real" spectrum of the source). In ISIS, the calculation of unfolded spectra is done in a model-independent way by using the response matrices, as opposed to XSPEC where the calculation of these spectra is performed through the distance of data points from the model. This calculation is less statistically robust, and is used only for display purposes. Model fitting and residuals are calculated in the usual way, by applying the response matrix to the model and comparing to the uncorrected detector counts. See more details in Nowak (2005).

13 http://pulsar.sternwarte.uni-erlangen.de/wilms/research/tbabs/
} 


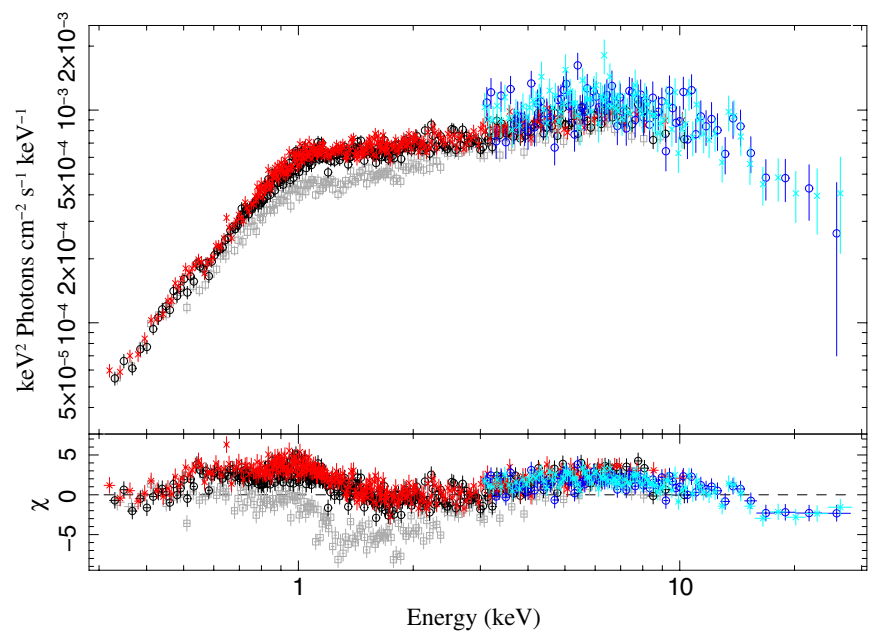

Figure 3. EPIC-pn and NUSTAR unfolded spectrum of NGC 1313 X-1 during the two observations, and residuals with respect to the best-fit absorbed power law in the XMM-Newton band. Black and red points are EPIC-pn data, and blue and cyan FPMA data. Circles indicate the first observation, crosses indicate the second. The spectrum shows a soft excess and a cutoff, as observed in this source when in its low-flux state. The archival 2006 October XMM-Newton observation is also plotted (gray squares) for comparison. Data are rebinned to 200 counts bin ${ }^{-1}$ (EPIC-pn) and 30 counts bin ${ }^{-1}$ (FPMA) for visual purposes. The spectral shape does not change significantly between the two observations and is qualitatively similar to the archival spectrum, even with a slightly higher flux. The slight misalignment between NuSTAR and XMM-Newton data is due to residual cross-calibration and possibly the non-perfect simultaneity of the observations.

(A color version of this figure is available in the online journal.)

free, ${ }^{14}$ to determine a cross-calibration constant that we fix for the subsequent fits. This takes into account residual crosscalibration between XMM-Newton and NUSTAR and the possible mismatches due to non-strictly simultaneous observations.

\section{2. $N G C 1313 X-1$}

Figure 3 shows an overview of the spectral features of $\mathrm{X}-1$. As can be seen in this plot, the spectrum did not change significantly between the two observations, either in the XMM-Newton or in the NUSTAR bands. We determined the cross-normalization constant between NuSTAR and XMMNewton data to be $1.20 \pm 0.06$ for FPMA and $1.29 \pm 0.07$ for FPMB in the first observation, and $1.18 \pm 0.06$ FPMA and $1.25 \pm 0.07$ FPMB in the second. We measure an absorbed $(0.3-10) \mathrm{keV}$ luminosity of $(6.3 \pm 1.0) \times 10^{39} \mathrm{erg} \mathrm{s}^{-1}$ in the first observation $\left(\sim 8.9 \times 10^{39}\right.$ unabsorbed, assuming the best-fit diskbb+cut of fpl model below) and $(6.6 \pm 1.0) \times 10^{39} \mathrm{erg} \mathrm{s}^{-1}$ in the second $\left(\sim 9 \times 10^{39}\right.$ unabsorbed $)$. The corresponding absorbed $0.3-30 \mathrm{keV}$ luminosities are $(8.1 \pm 1.0) \times 10^{39}$ and $(7.9 \pm 1.0) \times 10^{39} \mathrm{erg} \mathrm{s}^{-1}$, respectively. The spectral residuals with respect to the best-fit power law in the XMM-Newton band are qualitatively similar to the one reported from the 2006 October XMM-Newton observation (Dewangan et al. 2010), associated with the low-flux state of this source, as opposed to the higher states where the soft excess is less prominent, as also shown in Figure 3.

\subsubsection{Cutoff versus Reflection}

The first thing that becomes evident thanks to the NuSTAR data is that the spectrum shows a clear cutoff above $10 \mathrm{keV}$. As 14 The cross-calibration between pn and MOS $\{1,2\}$ is negligible with respect
to the one between pn and FPM in our data. we mentioned earlier, hints of this cutoff are present in $X M M$ Newton archival data of many ULXs, but this feature could be produced by a real cutoff or by relativistically smeared iron features. With the addition of NuSTAR data this degeneracy is broken. We fitted the data with three models: (1) a power law with exponential cutoff (XSPEC model cutoffpl) with and without an additional disk component modeled as a multicolor disk (MCD; diskbb; Mitsuda et al. 1984) ; (2) diskbb plus a Comptonization model (comptt; Titarchuk 1994) with the Comptonization seed photon temperature linked to the inner disk temperature for consistency; (3) a blurred reflection model obtained by convolving the reflionx table (Ross \& Fabian 2005) with a Laor profile (Laor 1991) provided by the convolution model kdblur2 to account for general relativity effects, following the method used by Walton et al. (2011a) and Caballero-Garcia \& Fabian (2010). See Table 2 for details.

While blurred reflection models and Comptonization/cutoff models yield similarly good fits in the XMM-Newton band alone, they predict a completely different behavior around and above $10 \mathrm{keV}$, as shown in Figure 4. In reflection models, by adding NUSTAR data we can find a decent nominal fit $\left(\chi^{2} /\right.$ dof $\sim 1.08$ in the first observation, 1.18 in the second; see Table 2), but it is mostly due to the large number of XMM-Newton spectral bins below $10 \mathrm{keV}$. From the residuals in Figure 4 it is clear that the description of the spectrum is inadequate around and above $10 \mathrm{keV}$. Even in the reflection-dominated regime where the power-law normalization is zero and the downturn produced by the broadened iron line is maximum, the downturn is not sufficient to account for the very deep cutoff seen in NuSTAR data, and the Compton "hump" produced by reflection clearly over predicts the spectrum above $10 \mathrm{keV}$.

\subsubsection{Comparison of Comptonization Models}

NUSTAR data enable us to obtain a much better constraint on the cutoff. This is shown in Figure 5, where the contour levels between $k T_{\mathrm{e}}$ and $\tau$ are shown with and without $N u S T A R$ data. It is clear from the contour plots that the addition of NuSTAR data improves the constraint considerably. An alternative way to show the poor constraint given by XMM-Newton data alone is to fix the electron temperature of comptt $\left(k T_{\mathrm{e}}\right)$ to $50 \mathrm{keV}$, and fit the data. If we take XMM-Newton data only, the fit deteriorates $\left(\Delta \chi^{2} \sim 20\right.$ in the first observation, $\sim 30$ in the second for $\Delta$ dof $=1$ ) but we can still recover an overall acceptable fit $\left(\chi^{2} /\right.$ dof $\sim 1.08$ in the first, 1.06 in the second) and even obtain a compatible value of the disk temperature (Table 2). XMMNewton response drops and data have only a few points around $10 \mathrm{keV}$, where the constraint on the cutoff is set, and small systematic errors in the instrument response can influence the fit. The difference between the two models disappears if one discards the last 20 bins of the spectrum. With the addition of NUSTAR data this is not true anymore, and in fact the fit deteriorates further $\left(\chi^{2} /\right.$ dof $\left.>1.2\right)$ and the disk temperature assumes incompatible values (see Table 2).

From Table 2 and Figure 4 it is also clear that the comptt model gives a slightly worse fit than the simple cutoffpl model, with a high-energy slope visibly steeper than what NUSTAR data show. This fact indicates that a single-temperature Comptonization model is probably not sufficient to describe the data. We make use of the optxagnf model (Done et al. 2012), which is a phenomenological model that represents the evolution of the dkbbfth model (Done \& Kubota 2006) often used for ULXs in the past (e.g., Gladstone et al. 2009; Walton et al. 2011a). optxagnf, originally developed for active 
Table 2

X-1: Best-fit Parameters for Some Common Spectral Models

\begin{tabular}{|c|c|c|c|c|c|}
\hline \multirow[b]{2}{*}{ Parameter } & \multirow[b]{2}{*}{ Unit } & \multicolumn{2}{|c|}{ EPIC-pn only } & \multicolumn{2}{|c|}{ pn, $\operatorname{MOS} 2, \operatorname{FPM}\{\mathrm{A}, \mathrm{B}\}$} \\
\hline & & Epoch 1 & Epoch 2 & Epoch 1 & Epoch 2 \\
\hline \multicolumn{6}{|c|}{ tbnew_feo*(cutoffpl) } \\
\hline $\begin{array}{l}n_{\mathrm{H}} \\
N_{\text {cut }} \\
\Gamma \\
E_{\text {cut }} \\
\chi^{2} / \text { dof } \\
\end{array}$ & $10^{22} \mathrm{~cm}^{-2}$ & $\begin{array}{c}0.267 \pm 0.006 \\
(7.9 \pm 0.1) \times 10^{-4} \\
2.00 \pm 0.02 \\
500_{-192}^{*} \\
1399 / 987\end{array}$ & $\begin{array}{c}0.267 \pm 0.006 \\
(8.3 \pm 0.1) \times 10^{-4} \\
2.02 \pm 0.02 \\
500_{-181}^{*} \\
1379 / 968\end{array}$ & $\begin{array}{c}0.265 \pm 0.006 \\
(7.9 \pm 0.1) \times 10^{-4} \\
1.97 \pm 0.03 \\
73_{-23}^{+62} \\
2699 / 1952\end{array}$ & $\begin{array}{c}0.257 \pm 0.006 \\
(8.2 \pm 0.1) \times 10^{-4} \\
1.93 \pm 0.03 \\
35_{-6}^{+9} \\
2707 / 1939\end{array}$ \\
\hline \multicolumn{6}{|c|}{ tbnew_feo*(diskbb+cutoffpl) } \\
\hline $\begin{array}{l}n_{\mathrm{H}} \\
N_{\mathrm{dbb}} \\
T_{\text {in }} \\
N_{\text {cut }} \\
\Gamma\end{array}$ & $10^{22} \mathrm{~cm}^{-2}$ & $\begin{array}{c}0.27 \pm 0.02 \\
11_{-3}^{+4} \\
0.30 \pm 0.02 \\
(4.2 \pm 0.6) \times 10^{-4} \\
1.1_{-0.3}^{+0.2}\end{array}$ & $\begin{array}{c}0.28 \pm 0.02 \\
12_{-3}^{+5} \\
0.29 \pm 0.02 \\
(4.7 \pm 0.6) \times 10^{-4} \\
1.2 \pm 0.2\end{array}$ & $\begin{array}{c}0.27 \pm 0.01 \\
10_{-2}^{+3} \\
0.31 \pm 0.01 \\
(4.1 \pm 0.3) \times 10^{-4} \\
1.0 \pm 0.1\end{array}$ & $\begin{array}{c}0.27 \pm 0.01 \\
10 \pm 2 \\
0.31 \pm 0.01 \\
(4.2 \pm 0.3) \times 10^{-4} \\
1.0 \pm 0.1\end{array}$ \\
\hline $\begin{array}{l}E_{\text {cut }} \\
\chi^{2} / \text { dof } \\
\end{array}$ & $\mathrm{keV}$ & $\begin{array}{c}6_{-2}^{+3} \\
1037 / 985 \\
\end{array}$ & $\begin{array}{c}9_{-3}^{+5} \\
1010 / 966 \\
\end{array}$ & $\begin{array}{c}6.3_{-0.7}^{+0.8} \\
1998 / 1950 \\
\end{array}$ & $\begin{array}{c}5.8 \pm 0.6 \\
1986 / 1937 \\
\end{array}$ \\
\hline \multicolumn{6}{|c|}{ tbnew_feo* $($ diskbb+comptt $)$} \\
\hline $\begin{array}{l}n_{\mathrm{H}} \\
N_{\mathrm{dbb}} \\
T_{\text {in }} \\
N_{\text {comp }} \\
k T_{\mathrm{e}} \\
\tau \\
\chi^{2} / \text { dof }\end{array}$ & $10^{22} \mathrm{~cm}^{-2}$ & $\begin{array}{c}0.27 \pm 0.02 \\
23_{-6}^{+9} \\
0.25 \pm 0.02 \\
(4.4 \pm 0.3) \times 10^{-4} \\
2.4_{-0.2}^{+0.3} \\
7.4 \pm 0.7 \\
1033 / 985\end{array}$ & $\begin{array}{c}0.28 \pm 0.02 \\
26_{-7}^{+10} \\
0.25 \pm 0.02 \\
(4.2 \pm 0.4) \times 10^{-4} \\
2.7_{-0.3}^{+0.5} \\
6.9 \pm 0.7 \\
1011 / 966\end{array}$ & $\begin{array}{c}0.28 \pm 0.01 \\
25_{-5}^{+7} \\
0.24 \pm 0.01 \\
(4.0 \pm 0.2) \times 10^{-4} \\
2.8_{-0.1}^{+0.2} \\
6.7 \pm 0.3 \\
2020 / 1950\end{array}$ & $\begin{array}{c}0.27 \pm 0.01 \\
25_{-5}^{+7} \\
0.24 \pm 0.01 \\
(4.3 \pm 0.2) \times 10^{-4} \\
2.7 \pm 0.1 \\
6.8 \pm 0.3 \\
2013 / 1937\end{array}$ \\
\hline \multicolumn{6}{|c|}{ tbnew_feo* $($ diskbb+comptt $)\left(k T_{\mathrm{e}}=50 \mathrm{keV}\right)$} \\
\hline $\begin{array}{l}n_{\mathrm{H}} \\
N_{\mathrm{dbb}}\end{array}$ & $10^{22} \mathrm{~cm}^{-2}$ & $\begin{array}{c}0.29 \pm 0.02 \\
38_{-9.6}^{+14}\end{array}$ & $\begin{array}{c}0.28 \pm 0.02 \\
36_{-9}^{+13}\end{array}$ & $\begin{array}{c}0.31 \pm 0.02 \\
71_{-18}^{+25}\end{array}$ & $\begin{array}{c}0.31 \pm 0.02 \\
98_{-28}^{+40}\end{array}$ \\
\hline $\begin{array}{l}T_{\text {in }} \\
N_{\text {comp }} \\
\tau \\
\chi^{2} / \text { dof } \\
\end{array}$ & $\mathrm{keV}$ & $\begin{array}{c}0.22 \pm 0.01 \\
(2.6 \pm 0.2) \times 10^{-5} \\
0.78_{-0.04}^{+0.05} \\
1069 / 986\end{array}$ & $\begin{array}{c}0.23 \pm 0.01 \\
(2.6 \pm 0.2) \times 10^{-5} \\
0.79 \pm 0.05 \\
1028 / 967\end{array}$ & $\begin{array}{c}0.19_{-0.01}^{+0.01} \\
(3.2 \pm 0.2) \times 10^{-5} \\
0.63 \pm 0.02 \\
2357 / 1951\end{array}$ & $\begin{array}{c}0.18 \pm 0.01 \\
(3.6 \pm 0.2) \times 10^{-5} \\
0.58 \pm 0.01 \\
2482 / 1938\end{array}$ \\
\hline \multicolumn{6}{|c|}{ tbnew_feo*(powerlaw+kdblur2 $(1$, reflionx $))$} \\
\hline $\begin{array}{l}n_{\mathrm{H}} \\
N_{\text {pow }}\end{array}$ & $10^{22} \mathrm{~cm}^{-2}$ & $\begin{array}{c}0.264_{-0.01}^{+0.003} \\
4.16_{-0.05}^{*} \times 10^{-4}\end{array}$ & $\begin{array}{c}0.265 \pm 0.009 \\
(6.4 \pm 0.3) \times 10^{-4} \\
\end{array}$ & $\begin{array}{c}0.282 \pm 0.007 \\
0^{*}\end{array}$ & $\begin{array}{c}0.282_{-0.007}^{+0.004} \\
0^{*} \\
5^{+0.2}\end{array}$ \\
\hline $\begin{array}{l}N_{\text {ref }} \\
A_{\mathrm{Fe}}\end{array}$ & & $\begin{array}{c}1.5_{-0.2}^{+0.3} \times 10^{-9} \\
20^{*}\end{array}$ & $\begin{array}{c}2_{-1}^{+2} \times 10^{-8} \\
5^{+8}\end{array}$ & $\begin{array}{c}(4.6 \pm 0.5) \times 10^{-9} \\
53^{+0.8}\end{array}$ & $\begin{array}{c}4.5_{-0.5}^{+0.2} \times 10^{-9} \\
5.9^{+0.6}\end{array}$ \\
\hline $\begin{array}{l}A_{\mathrm{Fe}} \\
\Gamma\end{array}$ & & $\begin{array}{c}20_{-1} \\
1.82_{-0.09}^{+0.03}\end{array}$ & $\begin{array}{c}\mathrm{J}_{-2} \\
1.85_{-0.02}^{+0.03}\end{array}$ & $\begin{array}{c}5.3_{-0.5} \\
1.65 \pm 0.04\end{array}$ & $\begin{array}{c}5.9_{-0.7}^{-0} \\
1.68_{-0.04}^{+0.02}\end{array}$ \\
\hline$X_{\mathrm{i}}$ & & $(3 \pm 1) \times 10^{3}$ & $\left(0.25_{-0.01}^{+0.50}\right) \times 10^{3}$ & $3.2_{-0.3}^{+0.4} \times 10^{3}$ & $3.3_{-0.2}^{+0.4} \times 10^{3}$ \\
\hline $\begin{array}{l}q \\
R_{\text {in }}\end{array}$ & & $\begin{array}{l}6_{-2}^{+3} \\
1_{*}^{+7}\end{array}$ & $\begin{array}{l}5_{-3}^{+5} \\
1_{*}^{+6}\end{array}$ & $\begin{array}{c}7 \pm 3 \\
1.32_{-0.08}^{+0.29}\end{array}$ & $\begin{array}{c}9.2_{-0.3}^{+0.8} \\
1.24 \pm 0.03\end{array}$ \\
\hline $\begin{array}{l}i \\
\chi^{2} / \text { dof }\end{array}$ & $\operatorname{deg}$ & $\begin{array}{c}71 \pm 5 \\
1011 / 973\end{array}$ & $\begin{array}{c}81_{-4}^{+5} \\
973.248 / 953\end{array}$ & $\begin{array}{c}68_{-16}^{+8} \\
2110 / 1877\end{array}$ & $\begin{array}{c}75_{-7}^{+1} \\
2281 / 1934\end{array}$ \\
\hline
\end{tabular}

Notes. All uncertainties refer to single-parameter $90 \%$ confidence limits.

*Values were fixed, or the parameter was unconstrained.

galactic nuclei (AGNs), tries to balance in a self-consistent way the optically thick emission from the disk, a low-temperature Comptonization component originating from the inner part of the disk, and a second, hot Comptonization component with cutoff above $100 \mathrm{keV}$ produced by a hot corona. With respect to the dkbbfth model, optxagnf adds a second Comptonizing component while maintaining the possibility of hiding the underlying disk emission below a corona that covers the disk and is powered by it. The latter was the reason dkbbfth was used in the past. Moreover, optxagnf has superior computational stability and a more convenient choice of parameters, using the expected mass, spin, and luminosity of the $\mathrm{BH}$ instead of a generic normalization parameter linked to the position of the inner disk (in fact, in this model the normalization factor should normally be frozen to 1 , but see below).

This model, however, has nine free parameters (mass, spin, luminosity, photon index and normalization of the hot Comptonizing component, optical thickness and temperature of the cold Comptonizing component, radius of this cold component, and outer radius of the disk) and therefore it is able to yield many different solutions for a given spectrum. We therefore restrict the parameter space by fixing some of them to reasonable values and discuss the results obtained with this approach, with the obvious associated caveats. A discussion of the full range 

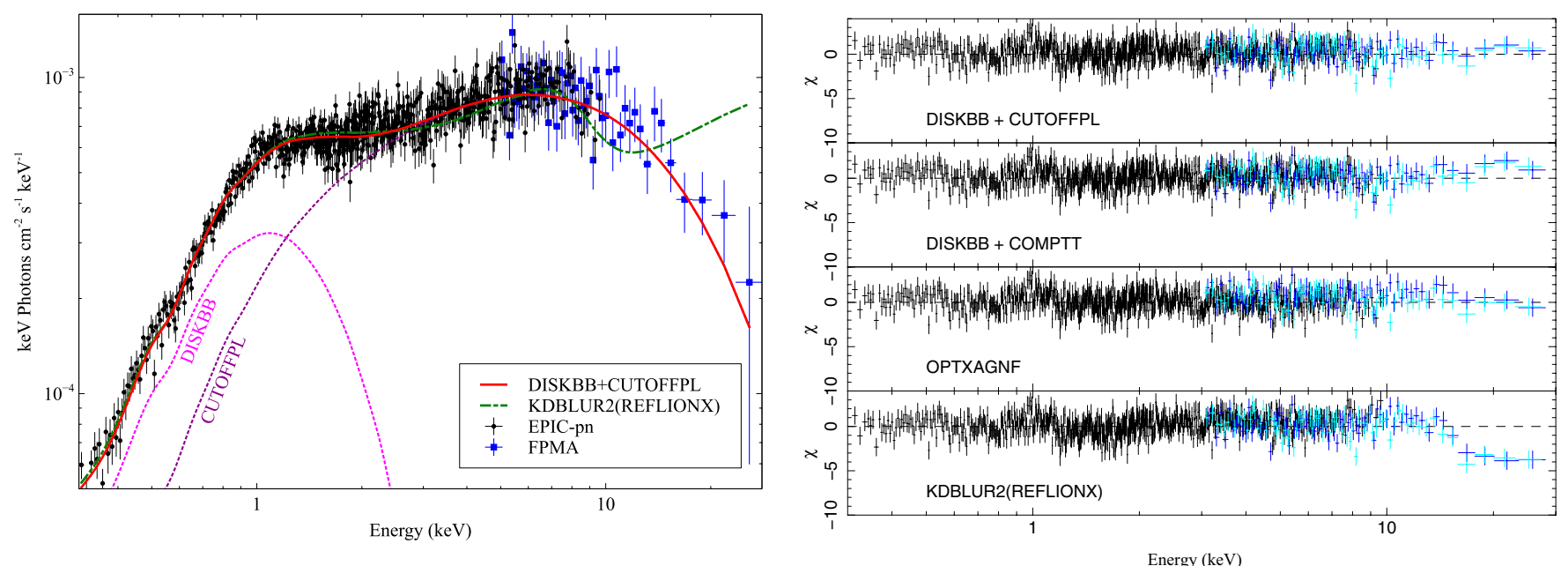

Figure 4. (Left) Unfolded spectrum of the second observation of X-1. Black circles represent EPIC-pn data, and blue squares represent FPMA data normalized with the cross-normalization constant. We superimpose the best-fit diskbb+cutoffpl model (red, solid), its single components (dashed), and the best-fit reflection model (green, dash-dotted). (Right) Residuals from a selection of models listed in Tables 2 and 3. Black is EPIC-pn, blue FPMA, and light blue FPMB. The models are calculated with all available detectors, but for clarity only pn data are plotted for XMM-Newton. Also for clarity, data have been rebinned to 30 counts bin $^{-1}$. The red model overplotted to the data in the spectrum shows the best fit with a reflection-dominated spectrum. Note that this model works very well in the XMM-Newton band, and the downturn produced by the iron line around $10 \mathrm{keV}$ is able to fit the cutoff below $10 \mathrm{keV}$, but the addition of NuSTAR data clearly rules it out.

(A color version of this figure is available in the online journal.)

of scenarios that this model can describe is beyond the scope of this work and will be discussed in a future paper.

Our NUSTAR data show an excess with respect to a singletemperature Comptonization model, but do not show the plateau at high energies that has been observed, for example, in the bright AGNs (Done et al. 2012). We therefore fix the power-law index of the hot electrons, $\Gamma$, to 2 , a typical value observed in $\mathrm{BH}$ power-law spectra, and we free only its normalization factor, $f_{\mathrm{PL}}$. We also fix the outer disk to $10^{5} R_{\mathrm{g}}$, the spin parameter $a$ to 0 and, as is prescribed in the documentation, the normalization factor to 1 . Because this model does not take into account the inclination and assumes an observing angle of $60^{\circ}$, and the norm is proportional to $\cos i / \cos 60^{\circ}$, we also fitted the data with the norm fixed to 2 (source seen face-on) to evaluate whether a change in this parameter could dramatically affect the results.

We summarize the best fit in this reduced parameter space in Table 3. In both observations this model yields an intriguing result: under the above assumptions $(a=0, \Gamma=2)$, and with both normalizations, the spectrum seems to be well described by a quite massive $\left(\sim 70-90 M_{\odot}\right) \mathrm{BH}$, accreting close to (or slightly above) Eddington, with a large corona reaching $\sim 60 R_{\mathrm{g}}$. The fraction of energy that is reprocessed from the hot part of the corona is about $60 \%$, while the rest is reprocessed by the cold and optically thick part. As expected, fixing the norm to 2 has the effect of lowering both the mass of the $\mathrm{BH}$ and the luminosity, but the rest of parameters do not change significantly.

All of the above models leave some residuals around $1 \mathrm{keV}$ and below. They appear very similar in all fits, indicating that they are independent from the particular continuum model used. Similar residuals are often observed in ULXs (see, e.g., Soria et al. 2004; Gonçalves \& Soria 2006; Gladstone et al. 2009; Caballero-Garcia \& Fabian 2010). We tested the improvement of the fit with the addition of a MEKAL component (Mewe \& Gronenschild 1981) to the cutoffpl and diskbb+cutoffpl models. We failed to obtain a good fit in the first case, while in the second we found a general improvement of the fit $\left(\Delta \chi^{2} \sim 50\right)$, with a MEKAL temperature of about $1 \mathrm{keV}$ and the abundances fixed to the standard values. As the diffuse emission from the

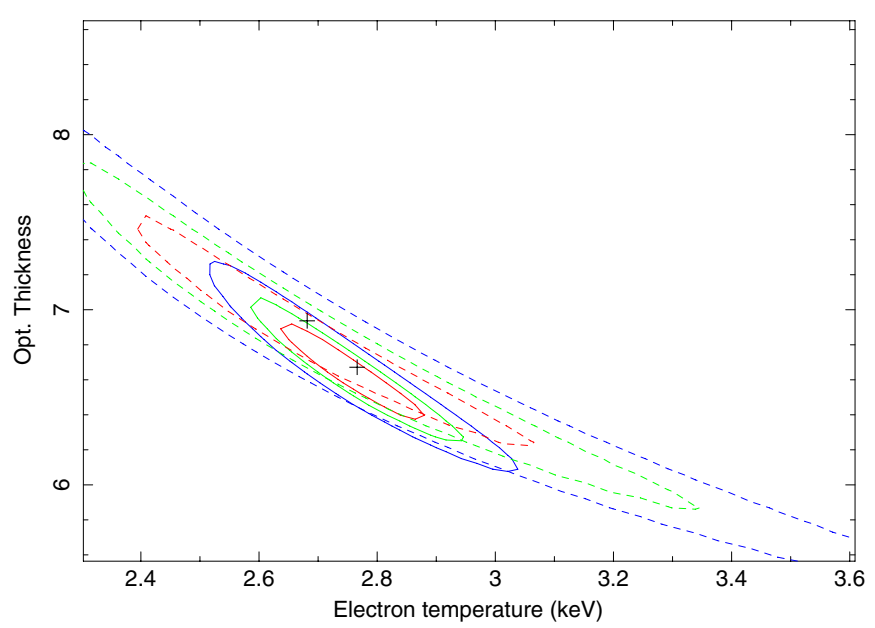

Figure 5. Confidence contours for the $k T_{\mathrm{e}}$ and $\tau$ parameters in the diskbb+comptt model fit for X-1, using only XMM-Newton data (dashed) and $X M M-N e w t o n$ and NUSTAR data (solid) of the second observation. The added value of NuSTAR data, when it comes to constraining the electron temperature, is evident.

(A color version of this figure is available in the online journal.)

NGC 1313 galaxy is negligible, this might indicate the presence of emission from a hot medium close to the source.

\section{3. $N G C 1313 X-2$}

As described in Section 3.1, we did not use NUSTAR data for the analysis of X-2. Figure 6 shows the shape of the XMMNewton spectrum of X-2 in the two epochs. The flux and overall shape of the spectrum changed considerably between the two observations, as did the timing behavior (see Section 5.4). We measure a $0.3-10 \mathrm{keV}$ absorbed luminosity of $(4.6 \pm 0.6) \times$ $10^{39} \mathrm{erg} \mathrm{s}^{-1}$ in the first epoch and $(2.2 \pm 0.6) \times 10^{39} \mathrm{erg} \mathrm{s}^{-1}$ in the second epoch.

Spectral fits with several models are presented in Table 4 . The spectrum is reasonably well described by an absorbed cutoff power law in both epochs, but the values of the spectral index 


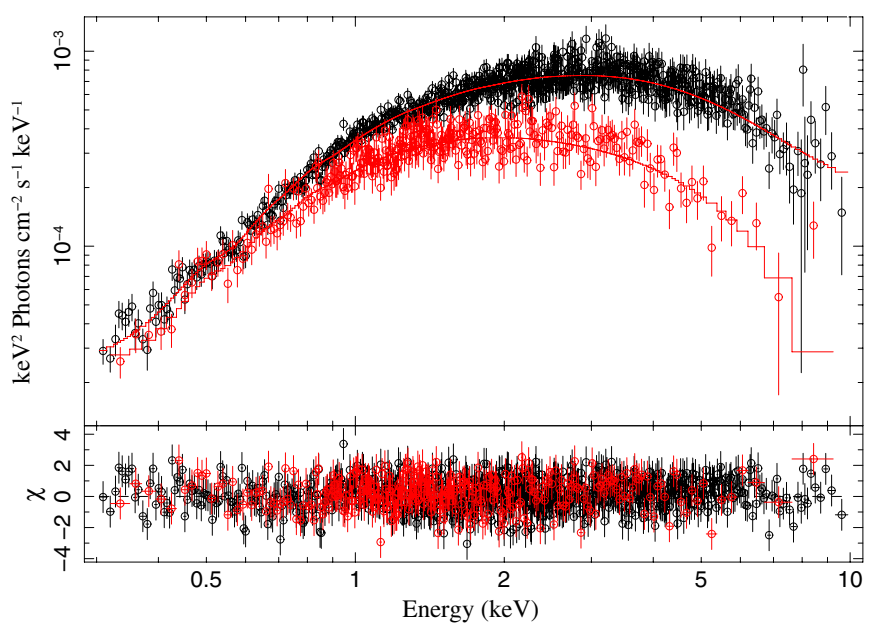

Figure 6. XMM-Newton unfolded spectra of NGC $1313 \mathrm{X}$-2 during the two observations. Black points are EPIC-pn and red points are EPIC-MOS1 data, due to the source being in the gap of pn in the second observation. The best-fit slim-disk model for the two observations with model ID set to 4 is superimposed. (A color version of this figure is available in the online journal.)

Table 3

X-1: Best-fit Parameters for optxagnf, with the Data from All Instruments

\begin{tabular}{lccc}
\hline \hline Parameter & Unit & Epoch 1 & Epoch 2 \\
\hline$n_{\mathrm{H}}$ & tbnew_feo*optxagnf & (norm fixed to 1) \\
$M$ & $10^{22}$ & $0.27_{-0.02}^{+0.01}$ & $0.26 \pm 0.01$ \\
$\log L / L_{\text {Edd }}$ & solar & $93_{-19}^{+17}$ & $91_{-14}^{+19}$ \\
$R_{\text {cor }}$ & & $-0.03_{-0.07}^{+0.1}$ & $-0.02_{-0.08}^{+0.07}$ \\
$k T_{\mathrm{e}}$ & $\mathrm{rg}$ & $66_{-5}^{+6}$ & $65 \pm 5$ \\
$\tau$ & $\mathrm{keV}$ & $2.0_{-0.3}^{+0.2}$ & $2.0 \pm 0.2$ \\
$f_{\mathrm{PL}}$ & & $10_{-1}^{+2}$ & $10_{-1.3}^{+1.5}$ \\
$\chi^{2} /$ dof & & $0.6 \pm 0.1$ & $0.58_{-0.12}^{+0.09}$ \\
\hline & & $1178 / 1190$ & $1327 / 1220$ \\
\hline$n_{\mathrm{H}}$ & tbnew_feo*optxagnf $($ norm fixed to 2$)$ \\
$M$ & $10^{22}$ & $0.27_{-0.01}^{+0.02}$ \\
$\log L / L_{\text {Edd }}$ & solar & $63_{-10}^{+14}$ & $0.26 \pm 0.01$ \\
$R_{\text {cor }}$ & & $-0.16_{-0.09}^{+0.08}$ & $65_{-10}^{+13}$ \\
$k T_{\mathrm{e}}$ & $\mathrm{rg}$ & $67_{-6}^{+5}$ & $-0.18 \pm 0.08$ \\
$\tau$ & $\mathrm{keV}$ & $2.0 \pm 0.2$ & $65_{-4}^{+5}$ \\
$f_{\mathrm{PL}}$ & & $11_{-2}^{+3}$ & $2.0 \pm 0.2$ \\
$\chi^{2} /$ dof & & $0.65_{-0.13}^{+0.09}$ & $10_{-1.3}^{+1.9}$ \\
\hline
\end{tabular}

Notes. All uncertainties refer to single-parameter $90 \%$ confidence limits. Note that for this model data were rebinned to 50 counts $\mathrm{bin}^{-1}$ for XMM-Newton in order to reduce computation times during error bar calculations.

(down to 0.9 in one case) are very different from what would be expected by Comptonization, the main process known to produce this kind of spectral shape, that instead yields spectral indices between 1.5 and 3 . The addition of a disk component to the cutoff power law barely improves the fit $\left(\Delta \chi^{2} \sim 3\right.$ in the first observation, and $\sim 6$ in the second, for 2 fewer degrees of freedom, dof). Similarly, a diskbb+comptt model does not improve the fit with respect to the cutoffpl model.

The spectral shape is clearly not well described by a standard MCD (XSPEC model diskbb), but it is well modeled by a so-called $p$-free disk (diskpbb; Mineshige et al. 1994; Kubota et al. 2005). When the accretion rate is high, it is expected that the structure of the disk deviates considerably from the
Table 4

X-2: Best-fit Parameters for Some Models, with the Data from XMM-Newton Only

\begin{tabular}{|c|c|c|c|}
\hline Parameter & Unit & Epoch 1 & Epoch 2 \\
\hline \multicolumn{4}{|c|}{ tbnew_feo*(cutoffpl) } \\
\hline$n_{\mathrm{H}}$ & $10^{22} \mathrm{~cm}^{-2}$ & $0.23 \pm 0.02$ & $0.29 \pm 0.02$ \\
\hline$N_{\text {cut }}$ & & $(7.6 \pm 0.2) \times 10^{-4}$ & $(5.8 \pm 0.2) \times 10^{-4}$ \\
\hline$\Gamma$ & & $0.9 \pm 0.1$ & $1.5 \pm 0.2$ \\
\hline$E_{\text {cut }}$ & $\mathrm{keV}$ & $2.5 \pm 0.2$ & $2.5_{-0.3}^{+0.4}$ \\
\hline$\chi^{2} /$ dof & & $776 / 833$ & $613 / 528$ \\
\hline \multicolumn{4}{|c|}{ tbnew_feo*(diskbb) } \\
\hline$n_{\mathrm{H}}$ & $10^{22} \mathrm{~cm}^{-2}$ & $0.153_{-0.005}^{+0.006}$ & $0.142 \pm 0.008$ \\
\hline$N_{\mathrm{dbb}}$ & & $0.056 \pm 0.003$ & $0.106 \pm 0.009$ \\
\hline$T_{\text {in }}$ & $\mathrm{keV}$ & $1.21 \pm 0.02$ & $0.86 \pm 0.02$ \\
\hline$\chi^{2} /$ dof & & $959 / 834$ & $833 / 529$ \\
\hline \multicolumn{4}{|c|}{ tbnew_feo*(diskpbb) } \\
\hline$n_{\mathrm{H}}$ & $10^{22} \mathrm{~cm}^{-2}$ & $0.27 \pm 0.02$ & $0.320_{-0.011}^{+0.008}$ \\
\hline$N_{\mathrm{dbb}}$ & & $0.010 \pm 0.002$ & $0.0059_{-0.0008}^{+0.0013}$ \\
\hline$T_{\text {in }}$ & $\mathrm{keV}$ & $1.56 \pm 0.06$ & $1.27 \pm 0.05$ \\
\hline$p$ & & $0.58 \pm 0.01$ & $0.500_{*}^{+0.006}$ \\
\hline$\chi^{2} / \mathrm{dof}$ & & $776 / 833$ & $611 / 528$ \\
\hline \multicolumn{4}{|c|}{ tbnew_feo*(diskbb+cutoffpl) } \\
\hline$n_{\mathrm{H}}$ & $10^{22} \mathrm{~cm}^{-2}$ & $0.22_{-0.02}^{+0.03}$ & $0.24_{-0.02}^{+0.04}$ \\
\hline$N_{\mathrm{dbb}}$ & & 0.7 (unconstr.) & $1.7_{-0.8}^{+1.6}$ \\
\hline$T_{\text {in }}$ & $\mathrm{keV}$ & $0.4_{-0.2}^{+0.1}$ & $0.38_{-0.10}^{+0.09}$ \\
\hline$N_{\text {cut }}$ & & $6_{-3}^{+2} \times 10^{-4}$ & $3_{-2}^{+3} \times 10^{-4}$ \\
\hline$\Gamma$ & & $0.5_{-1}^{+1.3}$ & $-0.3_{-1.2}^{+1.4}$ \\
\hline$E_{\text {cut }}$ & $\mathrm{keV}$ & $2.0 \pm 0.5$ & $1.2_{-0.2}^{+2.2}$ \\
\hline$\chi^{2} /$ dof & & $773 / 831$ & $607 / 526$ \\
\hline \multicolumn{4}{|c|}{ tbnew_feo*(diskbb+comptt) } \\
\hline$n_{\mathrm{H}}$ & $10^{22}$ & $0.183 \pm 0.008$ & $0.50_{-0.04}^{+0.05}$ \\
\hline$N_{\mathrm{dbb}}$ & & $0.20_{-0.06}^{+0.08}$ & $4_{-3}^{+12} \times 10^{4}$ \\
\hline$T_{\text {in }}$ & $\mathrm{keV}$ & $0.81 \pm 0.1$ & $0.07 \pm 0.01$ \\
\hline$N_{\text {comp }}$ & & $(1.2 \pm 0.4) \times 10^{-4}$ & $\left(2.6_{-0.4}^{+0.7}\right) \times 10^{-4}$ \\
\hline$k T_{\mathrm{e}}$ & $\mathrm{keV}$ & $2.0_{*}^{+0.1}$ & $2.0_{*}^{+0.1}$ \\
\hline$\tau$ & & $7.6_{-0.8}^{+1.7}$ & $5.0_{-0.2}^{+0.1}$ \\
\hline$\chi^{2} /$ dof & & $811 / 831$ & $661 / 526$ \\
\hline \multicolumn{4}{|c|}{ tbnew_feo*optxagnf } \\
\hline$n_{\mathrm{H}}$ & $10^{22} \mathrm{~cm}^{-2}$ & $0.18 \pm 0.01$ & $0.17_{-0.02}^{+0.04}$ \\
\hline$M$ & $M_{\odot}$ & $21_{-3}^{+4}$ & $32_{-9}^{+27}$ \\
\hline $\log L / L_{\mathrm{Edd}}$ & & $0.29_{-0.07}^{+0.06}$ & $-0.1_{-0.2}^{+0.1}$ \\
\hline$R_{\text {cor }}$ & rg & $39_{-16}^{+61}$ & $92_{-68}^{+8}$ \\
\hline$k T_{\mathrm{e}}$ & $\mathrm{keV}$ & $1.12_{-0.08}^{+0.13}$ & $0.87_{-0.07}^{+0.06}$ \\
\hline$\tau$ & & $16_{-3}^{+4}$ & $13_{-1}^{+7}$ \\
\hline$\chi^{2} / \mathrm{dof}$ & & $559 / 599$ & $443 / 422$ \\
\hline
\end{tabular}

Note. All uncertainties refer to single-parameter $90 \%$ confidence limits.

standard Shakura \& Sunyaev (1973) thin disk. In this model, the radial dependency of the disk temperature is parameterized with $T \propto r^{-p}$, where $p$ is different from the $3 / 4$ value used in the thin disk. The $p$-disk would recover the standard thin disk if $p=0.75$. For $p<0.75$ the temperature profile is affected by advection. At $p=0.5$, advection dominates and the disk is a so-called slim disk (Abramowicz et al. 1988; Watarai \& Fukue 1999).

The $p$-free model seems to yield a very good fit for both epochs, with values of $p$ very close to the slim disk regime. The amount of advection is closer to the slim disk regime in the fainter observation. This behavior has been reported for this and 
Table 5

X-2: Best-fit Parameters for X-2, with the slimdisk Model, and the Mass Tied between the Two Observations

\begin{tabular}{lccccccc}
\hline \hline model ID & $\alpha$ & $\begin{array}{c}n_{\mathrm{H}, 1} \\
\left(\mathrm{~cm}^{-2}\right)\end{array}$ & $\begin{array}{c}n_{\mathrm{H}, 2} \\
\left(\mathrm{~cm}^{-2}\right)\end{array}$ & $\begin{array}{c}M \\
\left(M_{\odot}\right)\end{array}$ & $\dot{M}_{1}\left(L_{\mathrm{Edd}} / c^{2}\right)$ & $\dot{M}_{2}\left(L_{\mathrm{Edd}} / c^{2}\right)$ & $\chi^{2} / \mathrm{dof}$ \\
\hline 7 & $0.0100_{*}^{+0.0006}$ & $0.296 \pm 0.006$ & $0.23 \pm 0.01$ & $21.4 \pm 0.8$ & $30 \pm 2$ & $12.2_{-0.3}^{+0.4}$ & $1521 / 1422$ \\
4 & $0.11_{-0.02}^{+0.04}$ & $0.265_{-0.006}^{+0.007}$ & $0.23 \pm 0.01$ & $36_{-1}^{+2}$ & $11.5_{-0.5}^{+0.4}$ & $5.1 \pm 0.2$ & $1456 / 1422$ \\
\hline
\end{tabular}

Notes. Subscripts 1 and 2 refer to the two observations. The mass was tied in the two observations. All uncertainties refer to single-parameter $90 \%$ confidence limits. modelID $=4$ means that we are modeling a slim disk plus Comptonization. With modelID $=7$ we are also adding relativistic corrections. As the grid of the slimdisk model was quite sparse, the errors on the parameters are typically inside the range between a value and the following in the grid. For this reason, one should use these uncertainties with some caution.

other ULXs in the past (Mizuno et al. 2007; Middleton et al. $2011 b$; Straub et al. 2013). The deviation of $p$ from 0.5 in the brightest observation might imply some reprocessing of the disk emission, for example by a corona.

The fact that these two observations have such different fluxes and spectral shapes gives us the opportunity to jointly fit the data with more physically motivated models that would be difficult to constrain with single observations, and try to obtain an estimate on the mass of the source.

With this goal in mind, we used an advanced slim disk model, implemented by Kawaguchi (2003, hereafter slimdisk). In this local XSPEC table model (used in the past for fitting ULX spectra, see, e.g., Vierdayanti et al. 2006; Godet et al. 2012) mass $M$ (in $M_{\odot}$ ) and accretion rate $\dot{M}$ are the only physical parameters. $\dot{M}$ is calculated in units of $L_{\mathrm{Edd}} / c^{2}$, where $L_{\text {Edd }}$ is the Eddington luminosity. Since $L \simeq \eta M c^{2}$, where $\eta$ is the efficiency, then the value of $\dot{M}$ corresponding to the Eddington luminosity is $1 / \eta \sim 16$, assuming the efficiency for a Schwarzschild BH calculated by using a pseudo-Newtonian potential (see, e.g., Ebisawa et al. 2003). Comptonization from a corona, gravitational redshift, and transverse Doppler effect are included self-consistently, but there is no observing angle dependence, as the source is assumed to be face-on. Ideally, this model provides a unique value of the mass given the mass accretion rate or vice versa. The choice of the spectral model (slim disk alone, with an additional thermal component or Comptonization, with or without relativistic effects) to include in the computation is done by switching the values of the modelID parameter. For our purposes, we are interested in the treatment of a slim disk with Comptonization, and with or without relativistic effects (i.e., using modelID equal to 4 or 7). The disk viscosity parameter $\alpha$, the only non-observable quantity, can also be set.

We tied almost all parameters of the model in the two epochs, leaving only the mass accretion rate $\dot{M}$ free to vary between them; we fixed the normalization to $(10 \mathrm{kpc} / d)^{2}=5.86 \times 10^{-6}$, assuming $d=4.13 \mathrm{Mpc}$.

We initially fixed the modelID to 7 , meaning that we used all corrections for gravity and Comptonization. We tried different values for the $\alpha$ parameter and found that the spectral shape was best described by $\alpha \sim 0.01$ (i.e., the lower limit of this table for the viscosity parameter). Even with these very restrictive assumptions, the model was able to fit the data quite well (Table 5).

By taking out the relativistic corrections, namely changing the modelID to 4 , we were again able to fit the data fairly well. In this case, there was need for a higher viscosity in order to reproduce the curvature of the spectrum. As a result, the measured values of the mass are higher, but always in the range of StMBHs.
In addition to the slimdisk model, we used the aforementioned optxagnf. We started by fitting the model to the single observations, similar to what was done for X-1 (best-fit results in Table 4), this time fixing the power-law index to 2.2 (by analogy with high accretion rate Seyfert galaxies) and the fraction of emission in the hot corona to 0.3. Then we fitted together the two observations. Given the complexity of this model, we used it to obtain estimates on the most likely source parameters by fixing the mass, the photon index of the hot electrons $\Gamma$, and the spin $a$ to discrete values $\left(10,30,60,90 M_{\odot}\right.$ for $M ; 1.8,2$, and 2.2 for $\Gamma ; 0$ and 0.998 for $a$ ) and freeing the fraction of hot power-law emission, the optical thickness, and the cold corona temperature. The norm was fixed to 1 and then to 2 , because of the arguments considered in Section 4.2.2. For norm=1, we found the best fit $\left(\chi^{2} /\right.$ dof $\left.=1.001\right)$ for $M=30 M_{\odot}$ and $\Gamma=2.2$. The fit with $M=10 M_{\odot}$ was always unacceptable $\left(\chi^{2} /\right.$ dof $\left.\gtrsim 1.7\right)$. For $M=60 M_{\odot}$ we could obtain an acceptable fit $\left(\chi^{2} /\right.$ dof $\left.\lesssim 1.05\right)$ only for $\Gamma=2$. In all other cases either the fit was worse or one or more parameters reached their hard limits, implying a non-ideal regime of the model. Varying the spin from 0 to 0.998 did not change the results dramatically, with $\tau, k T_{\mathrm{e}}$, and $f_{\mathrm{PL}}$ compensating for most of the change in spectral shape. As before, fixing the norm to 2 lowered the estimate on the mass, permitting us to obtain decent fit values $\left(\chi^{2} /\right.$ dof $\left.=1.06\right)$ for $M=10 M_{\odot}$ and $\Gamma=1.8-2$.

As a bottom line, the favored interpretation, from both optxagnf and slimdisk, seems to be a StMBH (up to $\sim 50 M_{\odot}$ ) accreting around Eddington, or transitioning between a super-Eddington and a sub-Eddington regime. The emission from the cold and thick corona given by optxagnf, extending over a large region of the inner disk, does not differ substantially from the bloated disk described in the slimdisk model, and it is thus not surprising that the two models produce similar results.

The same caveats discussed for X-1 apply here: this result is model-dependent and based on the assumptions we made about the parameters; only further investigation using more observations with different spectral states will tell if the constraints on the mass are robust.

\section{TIMING ANALYSIS}

We extracted filtered event lists for both ULXs from all data sets and produced light curves cleaned from gaps and periods of increased background activity. These data were then processed with the following timing analysis techniques.

\section{1. rms variability}

The first variability test we used on our data is the normalized excess variance test (Edelson et al. 1990; Vaughan et al. 2003). Let $S$ be the intrinsic variance of the source signal (as calculated 
from the light curve), $\sigma_{i}$ the standard error on the $i$ th bin of the light curve (calculated from Poissonian statistics), $\bar{\sigma}$ the mean standard error, and $\bar{I}$ the mean counts per bin in the light curve. The excess variance is then simply $S-\bar{\sigma}^{2}$; we normalize it as follows:

$$
F_{\mathrm{var}}=\sqrt{\frac{S-\bar{\sigma}^{2}}{\bar{I}^{2}}} .
$$

$F_{\text {var }}$ has the advantage of being a linear quantity and thus yields a measure of the intrinsic root mean square (rms) variability of the source. The error we quote is the one derived in Vaughan et al. (2003).

\subsection{Power Density Spectrum}

For each light curve, we extracted a power density spectrum (PDS), the normalized square modulus of the Fourier Transform (see van der Klis 1989 for an extensive review of the methods used in the following). We used the Leahy et al. (1983) normalization, so that the PDS has a white noise level of 2. Dead time effects can safely be ignored due to the very low count rates of the sources analyzed.

This timing analysis is very sensitive to data gaps in light curves, which produce low-frequency noise and spikes in the PDS. NUSTAR data, because of the very short orbital period of the satellite ( $\sim 90$ minutes) and the position of the source, have about $\sim 30$ minutes of occultation every orbit. Moreover, both XMM-Newton and NUSTAR data have other gaps due to, for example, the filtering of periods of high background activity. As a strategy in our analysis, we decided to fill gaps of very short length (several seconds) with white noise at the average count rate in the nearby $4000 \mathrm{~s}$ of data. We verified that, due to the very low count rate, this did not produce any spurious features in the spectrum. Data chunks with longer gaps, such as occultation periods, were simply ignored. This also limits the maximum length of single fast Fourier transforms for NuSTAR data to less than $\sim 1 \mathrm{hr}$, while there is no such constraint for XMM-Newton.

We used different rebinning factors in order to look for features with different spectral width. Following Barret \& Vaughan (2012), we used maximum-likelihood fitting to evaluate features in cases where the rebinning was not sufficient to attain the Gaussian regime. The maximum frequency investigated was $512 \mathrm{~Hz}$, to include possible high-frequency quasi-periodic oscillations (QPOs) as often observed in BH sources (see Remillard \& McClintock 2006; Belloni et al. 2012, for reviews). The minimum frequency was the inverse of the length of each analyzed chunk with no gaps. For NuSTAR this was limited to $\sim 0.3 \mathrm{mHz}$, while for XMM-Newton data $\sim 0.1 \mathrm{mHz}$.

\subsection{NGC $1313 X-1$}

The PDS of NGC $1313 \mathrm{X}-1$ is almost featureless. The Kolmogorov-Smirnov test, calculated from the light curve at different bin times, does not detect any variability and we find no significant detections of QPOs or low-frequency noise in the PDS. $F_{\text {var }}$ is consistent with 0 . This source historically showed variability only in its brighter states. Dewangan et al. (2010) studied the relation between variability and spectral states in X-1 and our timing results are, together with our spectral results (Section 4.2), compatible with what they call the "low-flux" state.

\section{4. $N G C 1313 X-2$}

The behavior of this source is quite interesting from the timing point of view. The results of the timing analysis are shown in Figure 7. The change in spectral shape observed in this source (Section 4.3) is also reflected in the timing properties. As Figure 7 shows, in the observation with higher flux the power spectrum shows low-frequency variability. The overall rms à la Vaughan et al. (2003) is $F_{\text {var }}=13.6(7) \%$ in the first observation, and very low, consistent with 0 , in the second. The PDS of the first observation shows a red-noise component but no significant QPOs. This variability increases with energy (see Figure 7). This is probably a hint of what models are more likely to describe the spectra. In fact, if a pure (slim) disk was responsible for this emission, the higher-energy variability would correspond to the part of the disk closest to the $\mathrm{BH}$, where variability timescales should be faster, surely well above $1 \mathrm{~Hz}$. But the PDS shows that this variability is mostly at low frequencies $(<1 \mathrm{mHz})$. This gives support to a geometry where instead the source of variability is the Comptonizing medium, whose contribution increases with energy and whose timescales are not necessarily linked to the timescales in the inner disk.

\section{DISCUSSION}

In this paper we present the first $N u S T A R+X M M-N e w t o n$ results on the two ULXs in NGC 1313. NuSTAR data have proven particularly useful for X-1, where the data above $10 \mathrm{keV}$ clearly show a cutoff that was not well constrained by XMMNewton (see Figure 5). In X-2, due to the soft spectrum and the unfavorable position in the field of view, NuSTAR data are not as decisive, but XMM-Newton data are sufficient to perform high-quality spectral and timing analysis below $10 \mathrm{keV}$.

\section{1. $X-1$}

The results obtained for this source thanks to $N U S T A R$ data represent a new landmark in the understanding of ULX physics. Before $N u S T A R$ was launched, ULX spectra had been studied in detail only below $10 \mathrm{keV}$. At least two different models were previously able to describe the spectral energy distribution: a reflection-dominated regime where the downturn is produced by a very strong and broadened iron line, and several combinations of MCDs (or other kinds of soft excess models) and lowtemperature Comptonized emission cutting off slightly below $10 \mathrm{keV}$.

We show also in this paper that, even with a $\sim 100 \mathrm{ks}$ pointing, the XMM-Newton spectrum alone is not sufficient to constrain the cutoff. Also using a reflection model gives a very nice description of the spectrum with low residuals and good $\chi^{2}$ in the $X M M-N e w t o n$ band. With $X M M-N e w t o n$ alone, the spectrum might describe a standard low-hard state of a quite massive $\mathrm{BH}$ with a strong Comptonized component from a hot and optically thin medium, a reflection dominated state where the underlying power law is not observable, or a soft disk component and a reprocessed component that cuts off around $10 \mathrm{keV}$.

The addition of NUSTAR data removes this degeneracy. In the NuSTAR band, the spectrum shows a very clear cutoff around $10 \mathrm{keV}$, similar in character to that expected from Comptonization by a cold, thick medium, but slightly less steep. The quality of NuSTAR data is such that we can put tight constraints on the cutoff energy as shown in Figure 5 and Table 2, and, as a result, the significance of a low-temperature disk component detected by XMM-Newton. The presence of a lowtemperature, optically thick Comptonized component suggests 

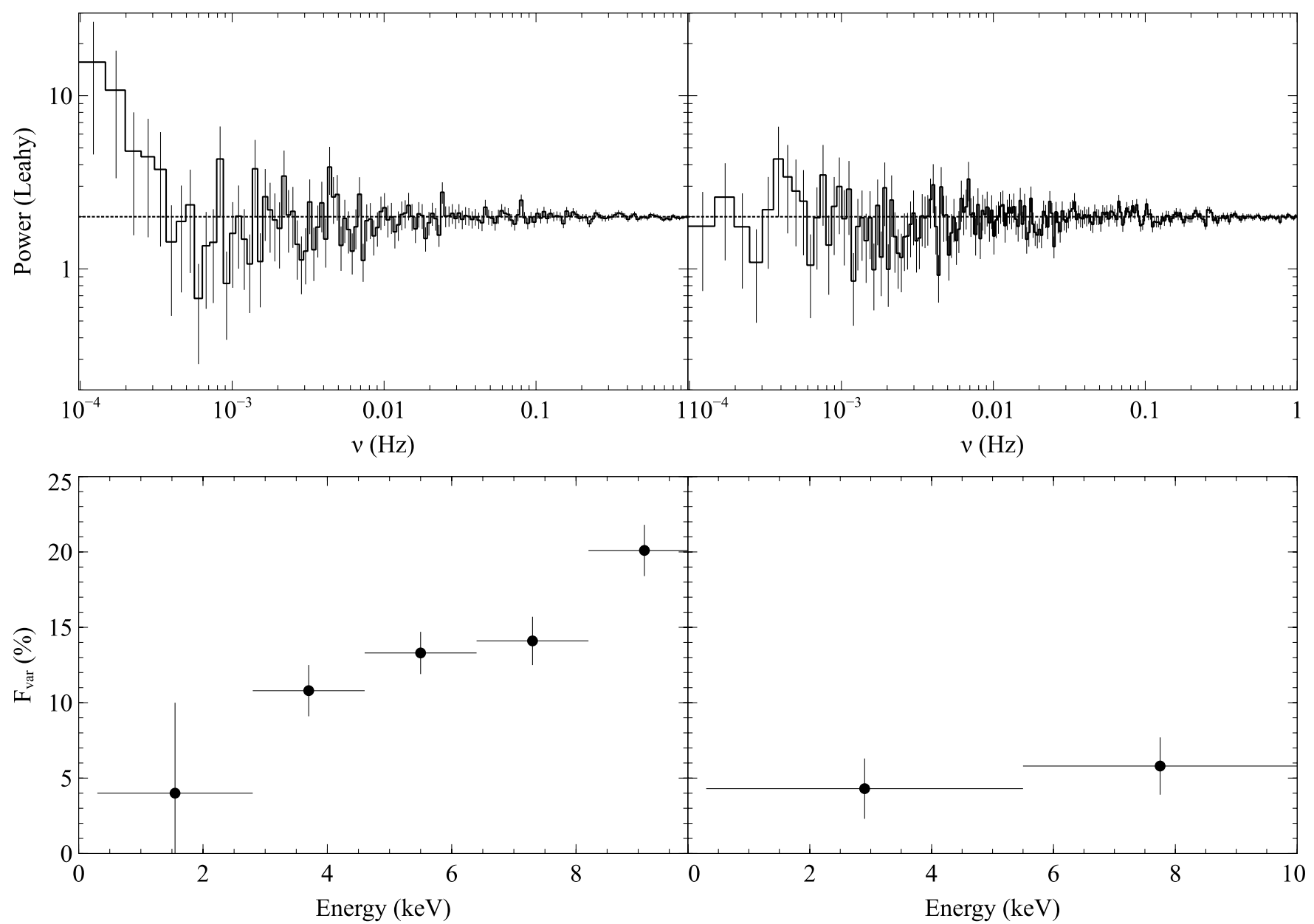

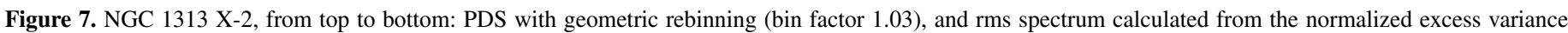

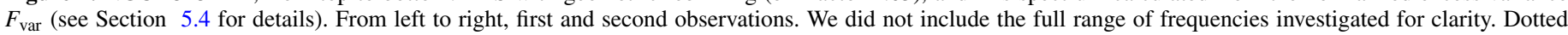

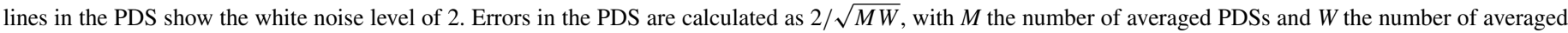

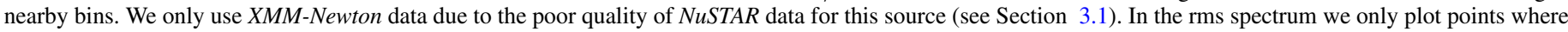
$F_{\text {var }}>0$. According to the PDS and the rms spectrum significant variability is only present in the first observation.

that we are observing accretion at high Eddington fractions that make the geometry of the system deviate substantially from the standard picture valid for lower luminosity BHs and confirms previous, albeit much less constraining, observations (e.g., Stobbart et al. 2006).

By going into more detail and fitting the optxagnf phenomenological model that includes a color-corrected MCD plus a two-component corona composed of a cold, optically thick medium and a second, hot and optically thin one, we obtain an interesting result: X-1 would be a quite massive StMBH of about 70-100 $M_{\odot}$, accreting close to Eddington, with a large, cold corona covering a significant part of the inner disk. This is in agreement with the lack of signatures of strong outflows that should be associated with highly super-Eddington accretion (see, e.g., Poutanen et al. 2007), such as photoionized bubbles (that are seen instead for other sources, e.g., in Pakull \& Mirioni 2002; Ramsey et al. 2006) or discrete atomic features in their high-energy spectra that could be associated with either iron emission or absorption from a wind (Walton et al. 2012). An alternative explanation is that these winds are not pointing toward the observer and the source is observed almost face-on (this would also agree with the lack of variability; see, e.g., Middleton et al. 2011a; Sutton et al. 2013).

To summarize, this source is clearly not accreting in a standard $\mathrm{BH}$ hard or soft state, as is shown by the absence of a power law and a spectrum not dominated by disk emission, and hence the high luminosity and the cold inner disk are not indicative of the mass. The spectral shape is instead well described by what is generally associated with accretion close to Eddington, that is an optically thick corona covering the inner part of the disk, and the energetics of the system points toward the high end of the StMBH population.

\section{2. $X-2$}

We caught a large spectral variation in X-2 that is extremely interesting both for the rapidity (one week) of the change and for its characteristics. The higher state is the one with the higher variability. This spectral behavior is reminiscent of the hard state of known BHs, where there is a linear correlation between rms variability and flux (see, e.g., Uttley \& McHardy 2001; McHardy 2010 for a review). Nonetheless, the shape of the spectra do not match the general picture of spectra in the hard state, where a prominent power-law component is usually present (Done et al. 2007). In our spectra the high-energy component drops off very quickly below or around $10 \mathrm{keV}$.

The spectrum of the source is instead well described by a StMBH with an advection-dominated disk, or slim disk, accreting around the Eddington limit. By linking the mass between the two observations and fitting the slimdisk model, 
and independently by using the optxagnf model, we are able to obtain an estimate of the mass of the $\mathrm{BH}$ around $25 M_{\odot}$ and a luminosity that is shifting from super- to sub-Eddington.

Even if Comptonization were not required by the spectrum, the fact that the most variability comes from higher energies, as shown in Figure 7, gives support to models including this component. A simple slim disk would be able to yield this variation of rms with energy but we would expect the highest energies to be produced in the region of the disk closer to the $\mathrm{BH}$, where variability is faster. This is in contradiction to the very low frequencies we observe in the PDS, and makes us favor an interpretation where variability comes instead from the corona, whose relative contribution to the spectrum is more important at high energies.

M.B. wishes to acknowledge the support from the Centre National d'Études Spatiales (CNES). This work was supported under NASA Contract No. NNG08FD60C, and made use of data from the NUSTAR mission, a project led by the California Institute of Technology, managed by the Jet Propulsion Laboratory, and funded by the National Aeronautics and Space Administration. We thank the NuSTAR Operations, Software, and Calibration teams for support with the execution and analysis of these observations. This research has made use of the NuSTAR Data Analysis Software (NuSTARDAS) jointly developed by the ASI Science Data Center (ASDC, Italy) and the California Institute of Technology (USA). This work also makes use of observations obtained with XMM-Newton, an ESA science mission with instruments and contributions directly funded by ESA Member States and NASA, and of observations made by the Chandra $X$-ray Observatory. For timing analysis and plotting, a set of Python codes making use of the NumPy and Scipy libraries was used. For some plots, we used the Veusz software. The authors wish to thank Olivier Godet and Chris Done for interesting discussions, and the referee Matt Middleton, whose comments and suggestions substantively improved the quality of the manuscript.

\section{REFERENCES}

Abramowicz, M. A., Czerny, B., Lasota, J. P., \& Szuszkiewicz, E. 1988, ApJ, 332,646

Arnaud, K. A. 1996, adass, 101, 17

Barret, D., \& Vaughan, S. 2012, ApJ, 746, 131

Belloni, T. M., Sanna, A., \& Mendez, M. 2012, MNRAS, 426, 1701

Caballero-Garcia, M. D., \& Fabian, A. C. 2010, MNRAS, 402, 2559

Dewangan, G. C., Misra, R., Rao, A. R., \& Griffiths, R. E. 2010, MNRAS, 407, 291

Done, C., Davis, S. W., Jin, C., Blaes, O., \& Ward, M. 2012, MNRAS, 420, 1848

Done, C., Gierliński, M., \& Kubota, A. 2007, A\&ARv, 15, 1

Done, C., \& Kubota, A. 2006, MNRAS, 371, 1216

Ebisawa, K., Życki, P., Kubota, A., Mizuno, T., \& Watarai, K.-y. 2003, ApJ, 597,780

Edelson, R. A., Krolik, J. H., \& Pike, G. F. 1990, ApJ, 359, 86

Farrell, S. A., Webb, N. A., Barret, D., Godet, O., \& Rodrigues, J. M. 2009, Natur, 460, 73

Feng, H., \& Kaaret, P. 2006, ApJL, 650, L75

Feng, H., \& Soria, R. 2011, NewAR, 55, 166

Gladstone, J. C., Roberts, T. P., \& Done, C. 2009, MNRAS, 397, 1836

Gladstone, J. C., Roberts, T. P., \& Done, C. 2011, AN, 332, 345

Godet, O., Plazolles, B., Kawaguchi, T., et al. 2012, ApJ, 752, 34
Gonçalves, A. C., \& Soria, R. 2006, MNRAS, 371, 673

Harrison, F. A., Craig, W. W., Christensen, F. E., et al. 2013, ApJ, 770, 103

Heil, L. M., Vaughan, S., \& Roberts, T. P. 2009, MNRAS, 397, 1061

Houck, J. C., \& Denicola, L. A. 2000, adass, 216, 591

Jansen, F., Lumb, D., Altieri, B., et al. 2001, A\&A, 365, L1

Joye, W. A., \& Mandel, E. 2003, adass, 295, 489

Kajava, J. J. E., \& Poutanen, J. 2009, MNRAS, 398, 1450

Kalberla, P. M. W., Burton, W. B., Hartmann, D., et al. 2005, A\&A, 440, 775

Kawaguchi, T. 2003, ApJ, 593, 69

King, A. 2004, NuPhS, 132, 376

Kubota, A., Ebisawa, K., Makishima, K., \& Nakazawa, K. 2005, ApJ, 631,1062

Laor, A. 1991, ApJ, 376, 90

Leahy, D. A., Darbro, W., Elsner, R. F., et al. 1983, ApJ, 266, 160

McHardy, I. 2010, The Jet Paradigm (Lecture Notes in Physics, Vol. 794; Berlin: Springer), 203

Méndez, B., Davis, M., Moustakas, J., et al. 2002, AJ, 124, 213

Mewe, R., \& Gronenschild, E. H. B. M. 1981, A\&AS, 45, 11

Middleton, M. J., Miller-Jones, J. C. A., Markoff, S., et al. 2013, Natur, 493, 187

Middleton, M. J., Roberts, T. P., Done, C., \& Jackson, F. E. 2011a, MNRAS, 411,644

Middleton, M. J., Sutton, A. D., \& Roberts, T. P. 2011b, MNRAS, 417, 464

Middleton, M. J., Sutton, A. D., Roberts, T. P., Jackson, F. E., \& Done, C. 2012, MNRAS, 420, 2969

Miller, J. M., Fabbiano, G., Miller, M. C., \& Fabian, A. C. 2003, ApJL, 585, L37

Miller, J. M., Fabian, A. C., \& Miller, M. C. 2004, ApJL, 614, L117

Miller, J. M., Walton, D. J., King, A. L., et al. 2013, ApJL, 776, L36

Mineshige, S., Hirano, A., Kitamoto, S., Yamada, T. T., \& Fukue, J. 1994, ApJ, 426, 308

Mitsuda, K., Bautz, M., Inoue, H., et al. 2007, PASJ, 59, 1

Mitsuda, K., Inoue, H., Koyama, K., et al. 1984, PASJ, 36, 741

Mizuno, T., Miyawaki, R., Ebisawa, K., et al. 2007, PASJ, 59, 257

Nowak, M. 2005, Ap\&SS, 300, 159

Pakull, M. W., \& Mirioni, L. 2002, in Proc. New Visions of the X-Ray Universe in the XMM-Newton and Chandra Era (Noordwijk: ESTEC), arXiv:astro-ph/0202488

Pintore, F., \& Zampieri, L. 2011, AN, 332, 337

Pintore, F., \& Zampieri, L. 2012, MNRAS, 420, 1107

Poutanen, J., Lipunova, G., Fabrika, S., Butkevich, A. G., \& Abolmasov, P. 2007, MNRAS, 377, 1187

Ramsey, C. J., Williams, R. M., Gruendl, R. A., et al. 2006, ApJ, 641, 241

Remillard, R. A., \& McClintock, J. E. 2006, ARA\&A, 44, 49

Roberts, T. P. 2007, Ap\&SS, 311, 203

Ross, R. R., \& Fabian, A. C. 2005, MNRAS, 358, 211

Shakura, N. I., \& Sunyaev, R. A. 1973, A\&A, 24, 337

Soria, R., Motch, C., Read, A. M., \& Stevens, I. R. 2004, A\&A, 423, 955

Stobbart, A.-M., Roberts, T. P., \& Wilms, J. 2006, MNRAS, 368, 397

Straub, O., Done, C., \& Middleton, M. 2013, A\&A, 553, 61

Sutton, A. D., Roberts, T. P., \& Middleton, M. J. 2013, MNRAS, 435, 1758

Swartz, D. A., Ghosh, K. K., Tennant, A. F., \& Wu, K. 2004, ApJS, 154, 519

Titarchuk, L. 1994, ApJ, 434, 570

Uttley, P., \& McHardy, I. M. 2001, MNRAS, 323, L26

van der Klis, M. 1989, in Timing Neutron Stars: Proceedings of the NATO Advanced Study Institute on Timing Neutron Stars held April 4-15, ed. H. Ogelman \& E. J. P. van den Heuvel (Dordrecht: Kluwer), 27

Vaughan, S., Edelson, R., Warwick, R. S., \& Uttley, P. 2003, MNRAS, 345,1271

Verner, D. A., Ferland, G. J., Korista, K. T., \& Yakovlev, D. G. 1996, ApJ, 465,487

Vierdayanti, K., Mineshige, S., Ebisawa, K., \& Kawaguchi, T. 2006, PASJ, 58,915

Walton, D. J., Gladstone, J. C., Roberts, T. P., et al. 2011a, MNRAS, 414, 1011

Walton, D. J., Miller, J. M., Reis, R. C., \& Fabian, A. C. 2012, MNRAS, 426,473

Walton, D. J., Roberts, T. P., Mateos, S., \& Heard, V. 2011b, MNRAS, 416, 1844

Watarai, K.-y., \& Fukue, J. 1999, PASJ, 51, 725

Weisskopf, M. C., Brinkman, B., Canizares, C., et al. 2002, PASP, 114, 1

Wilms, J., Allen, A., \& McCray, R. 2000, ApJ, 542, 914 